\title{
Efeitos da laserterapia de baixa intensidade nos mecanismos celulares e moleculares em procedimentos odontológicos: revisão integrativa
}

\author{
Effects of low-level laser therapy on cellular and molecular mechanisms in dental \\ procedures: an integrative review
}
Efectos de la terapia láser de baja intensidad en mecanismos celulares y moleculares en procedimientos dentales: revisión integrativa

José Eriverton Sousa Nogueira ${ }^{1 *}$, Ana Clara Araújo Fernandes ${ }^{1}$, Zildenilson da Silva Sousa ${ }^{1}$, Ana Beatriz Rodrigues de Mesquita ${ }^{1}$, Jady Júlia Patrício de Sousa1, José Auberlanio Lima Rodrigues $^{2}$, Perilo Marques Chaves Júnior ${ }^{1}$, Natanael Carvalho de Mesquita ${ }^{1}$, Maria Ocileide de Araújo ${ }^{3}$, Thaylon Menezes Ferreira da Silva ${ }^{1}$.

\section{RESUMO}

Objetivo: Realizar uma revisão integrativa sobre os efeitos celulares e moleculares da laserterapia de baixa intensidade (LBI) em procedimentos odontológicos. Métodos: Os descritores "Inflammation Mediators", "LowLevel Light Therapy", "Dentistry", "Biomarkers", "Clinical Trial", "Drug Evaluation" e "Preclinical" foram aplicados no Sistema Online de Busca e Análise de Literatura Médica (MEDLINE) via National Library of Medicine (PubMed), Science Direct e Google Scholar, interligados através de "AND/OR". O período de 10 anos (2011 a 2021) foi utilizado sendo encontrados um total de 130 estudos que após análise apenas 15 foram incluídos. Resultados: A literatura evidencia essa terapia como promissora para diversos problemas que afetam a cavidade oral, proporcionando efeitos regenerativos no processo de cicatrização, efeitos antiinflamatórios, reduções de quadros álgicos, desempenhando ainda um papel de suma importância na modulação de citocinas e fatores de crescimento celular em procedimentos odontológicos. Os níveis de interleucina 1 (IL-1 $\beta$ ) foram variáveis em associação a LBI, os níveis de substancias $P$ e matrix metalloproteinase-8 (MMP-8) foram reduzidos nos grupos avaliados. Considerações finais: A LBI pode promover respostas clínicas positivas na odontologia visto a ação dos mediadores envolvidos nestes processos regenerativos, contudo, devido à escassez de estudos em alguns campos da odontologia, faz-se necessário mais pesquisas na área.

Palavras-chave: Terapia com luz de baixa intensidade, Biomarcadores, Mecanismos moleculares de ação farmacológica, Odontologia.

\begin{abstract}
Objective: To carry out an integrative review of the cellular and molecular effects of low-intensity laser therapy (LLL) in dental procedures. Methods: The descriptors "Inflammation Mediators", "Low-Level Light Therapy", "Dentistry", "Biomarkers", "Clinical Trial", "Drug Evaluation" and "Preclinical" were applied to the Online Search and Analysis of Medical Literature System (MEDLINE) via National Library of Medicine (PubMed), Science Direct and Google Scholar, linked through "AND/OR". The 10-year period (2011 to 2021) was used and a total of 130 studies were found, which after analysis only 15 were included. Results: The literature shows this therapy as promising for several problems that affect the oral cavity, providing regenerative effects in the
\end{abstract}

${ }^{1}$ Centro Universitário Maurício de Nassau (UNINASSAU), Fortaleza - CE.

*E-mail: erivertonsousa2@gmail.com

2 Faculdade de Ensino e Cultura do Ceará (FAECE), Fortaleza - CE.

3 Universidade Estadual do Ceará (UECE), Tauá - CE. 
healing process, anti-inflammatory effects, reduction of pain conditions, also playing an extremely important role in the modulation of cytokines and factors of cell growth in dental procedures. The levels of interleukin 1 $(\mathrm{IL}-1 \beta)$ were variable in association with LLLT, the levels of substances $\mathrm{P}$ and matrix metalloproteinase-8 (MMP-8) were reduced in the groups evaluated. Final considerations: LLLT can promote positive clinical responses in dentistry due to the action of mediators involved in these regenerative processes, however, due to the scarcity of studies in some fields of dentistry, further research is needed in the area.

Keywords: Low intensity light therapy, Biomarkers, Molecular mechanisms of pharmacological action, Dentistry.

\section{RESUMEN}

Objetivo: Realizar una revisión integradora de los efectos celulares y moleculares de la terapia con láser de baja intensidad (LLL) en procedimientos dentales. Métodos: Los descriptores "Mediadores de inflamación", "Terapia de luz de bajo nivel", "Odontología", "Biomarcadores", "Ensayo clínico", "Evaluación de fármacos" y "Preclínico" se aplicaron en el Sistema de Búsqueda y Análisis Online de Literatura Médica (MEDLINE) a través de la Biblioteca Nacional de Medicina (PubMed), Science Direct y Google Scholar, vinculados a través de "Y / O". Se utilizó el período de 10 años (2011 a 2021) y se encontraron un total de 130 estudios, que luego del análisis solo se incluyeron 15. Resultados: La literatura muestra esta terapia como prometedora para varios problemas que afectan la cavidad oral, proporcionando efectos regenerativos en el proceso de curación, efectos antiinflamatorios, reducción de las condiciones de dolor, además de jugar un papel sumamente importante en la modulación de citocinas y factores de crecimiento celular en procedimientos dentales. Los niveles de interleucina 1 (IL-1 $\beta$ ) fueron variables en asociación con LLLT, los niveles de sustancias $\mathrm{P}$ y metaloproteinasa de matriz-8 (MMP-8) se redujeron en los grupos evaluados. Consideraciones finales: LLLT puede promover respuestas clínicas positivas en odontología debido a la acción de mediadores involucrados en estos procesos regenerativos, sin embargo, debido a la escasez de estudios en algunos campos de la odontología, se necesita más investigación en el área.

Palabras clave: Terapia de luz de baja intensidad, Biomarcadores, Mecanismos moleculares de acción farmacológica, Odontología.

\section{INTRODUÇÃO}

A laserterapia de baixa intensidade (LBI) é uma fonte de luz que possui inúmeras aplicações em todos os campos da ciência, tornando-se uma alternativa interessante em diversos procedimentos tradicionais realizados na odontologia (SRIVASTAVA V, et al., 2014). Essa terapia é considerada não invasiva e de poucos efeitos colaterais, sendo produtora de respostas imediatas como a redução da dor, e respostas tardias, envolvendo biossíntese de colágeno (SOUZA ACOC, et al., 2020).

É caracterizada por sua ação analgésica e estimulante em cicatrizações, auxiliando na redução de processos inflamatórios, promovendo por consequente o restabelecimento da função do aparelho mastigatório (SOUZA ACOC, et al., 2020; JÚNIOR C, et al., 2013). Esses efeitos ao serem estimulados pela LBI podem gerar o processo de modulação dos mediadores de inflamação celular (ASSIS TO, et al., 2012), elevando a produção de adenosina trifosfato e ativando espécies reagentes de oxigênio intracelular, no qual melhoram as respostas de possíveis inflamações no organismo, além de possuir a capacidade de promover a reparação tecidual (SANT'ANNA EF, et al., 2017).

Os lasers mais comuns usados na odontologia atualmente são o de dióxido de carbono (CO2), neodímio ítrio-alumínio-granada (Nd: YAG), érbio ítrio-alumínio-granada (Er: YAG), érbio cristal de granada-ítrioescândio-gálio sensibilizado com cromo (Er, Cr: YSGG) e diodo (SRIVASTAVA V, et al., 2014). É composto de três partes principais: uma fonte de energia, um meio de laser ativo e uma cavidade óptica ou ressonador e a energia produzida pode ter quatro interações diferentes com um tecido alvo, a saber, reflexão, absorção, espalhamento e transmissão (SRIVASTAVA V, et al., 2014). Embora todas as interações ocorram simultaneamente, é a interação de absorção que é importante na odontologia (SRIVASTAVA V, et al., 2014). 
Cada um produz um comprimento de onda de luz diferente e é genericamente denominado de acordo com o meio ativo contido no dispositivo, uma vez que nenhum comprimento de onda de laser único pode ser usado para tratar todas as doenças dentárias de maneira ideal (SRIVASTAVA V, et al., 2014). Os lasers possuem uma ação biomoduladora importante no processo de reparação tecidual oral, agindo de maneira terapêutica, sendo comumente utilizados em várias áreas das ciências da saúde, em especial a odontologia com uma maior prevalência na literatura científica (JÚNIOR C, et al., 2013). A LBI atua através da bioestimulação, alterando as funções celulares e teciduais, operando nas mitocôndrias e fazendo com que produzam mais trifosfato de adenosina, inibindo a via da ciclooxigenase, aumentando os níveis de serotonina, endorfina e diminuindo os níveis de prostaglandina e interleucina beta (SOUZA ACOC, et al., 2020).

Essa fermenta pode ser dividida em comprimento de onda utilizado, potência de saída, frequência, tempo de irradiação, intensidade da energia, intervalo e dosagem de tratamento escolhido pelo cirurgião-dentista durante sua aplicação (JÚNIOR C, et al., 2013). Para operar esses parâmetros clínicos, é fundamental que o profissional possua conhecimento do tipo de laser utilizado em diferentes campos em que essa luz irá atravessar até atingir o seu alvo, para que assim seja possível promover sua eficácia terapêutica naquele determinado procedimento, visto que a energia absorvida pelo tecido é a principal causa da ação reparadora no processo celular (SOUZA NHC, et al., 2014).

Para Souza NHC, et al. (2014), ainda há muito a ser estudado sobre a LBI visando entender de uma maneira mais analítica os efeitos dessa ferramenta nesses processos celulares e moleculares, além da importância de como evidenciar os parâmetros dosimétricos ideais de modulação nessa ação de reparo tecidual. Diante disso, o objetivo do presente estudo foi descrever, por meio de uma revisão integrativa, o efeito da laserterapia de baixa intensidade na odontologia e sua ação moduladora nos mecanismos celulares e moleculares.

\section{MÉTODOS}

Trata-se de uma revisão integrativa da literatura realizada entre janeiro e maio de 2021. Para sua elaboração foram definidas seis etapas operacionais, descritas por Souza MT, et al. (2010): 1) estipulação do problema da pesquisa; 2) busca e amostragem da literatura, com definição dos critérios de elegibilidade a serem adotados; 3) escolha das informações a serem extraídas, através de um instrumento previamente selecionado pelos autores, o software Microsoft Excel 2013; 4) análise dos estudos incluído; 5) discussão dos resultados, através da comparação dos dados obtidos com aqueles encontrados na literatura; 6) apresentação da revisão integrativa, com a finalização da escrita, e criação de quadros e tabelas que permitam a compreensão dos achados.

Para o direcionamento do estudo, elaborou-se a seguinte pergunta norteadora: "Quais processos celulares e moleculares a laserterapia de baixa intensidade promove em procedimentos odontológicos?". Para tanto, utilizou-se o acrônimo PICOS: paciente (Camundongos e humanos), intervenção (Submetidas a estudos odontológicos), comparação (tratamento com LBI), desfecho (processos celulares e moleculares) e desenho do estudo (ensaios clínicos e pré-clínicos).

Logo após, utilizou-se de três estratégias de pesquisa realizadas distintamente. Na primeira etapa, aplicouse dos descritores cadastrados nos descritores em ciências da saúde (DeCS): "Biomarkers", "Low-Level Light Therapy" e "Dentistry", na Medical Literature Analysis and Retrieval System Online (MEDLINE) através da National Library of Medicine (PubMed), interligados entre si através do operador booleano "and". Aplicou-se um filtro de tempo para os últimos 10 anos (2011 a 2021), sendo encontrados um total de 09 estudos e selecionados 06 .

Na segunda etapa de pesquisa bibliográfica, além dos descritores e período já utilizados anteriormente na PubMed, incluiu-se o descritor "Inflammation Mediators", interligado pelo mesmo operador booleano "and" na base de dados da Science Direct, sendo encontrados posteriormente um total de 116 artigos em um período de 10 anos (2011 a 2021), que após análise 05 estudos potencialmente relevantes para a temática abordada foram selecionados. 
Na terceira etapa da pesquisa, a literatura cinzenta (Google Scholar) foi utilizada visando obter mais dados da literatura. Assim, com os mesmos 4 descritores utilizados anteriormente na PubMed e Science Direct incluise os descritores "Clinical Trial" AND/OR "Drug Evaluation, Preclinical". Encontrou-se um total de 326 estudos que após análise foram selecionados 04 . O período aplicado foi de 5 anos (2016 a 2021).

Esta revisão foi elaborada de acordo com os seguintes critérios de inclusão: o desenho da pesquisa deveria conter apenas ensaios clínicos e pré-clínicos, sem restrição de idioma, publicados nos últimos 10 anos (2011 a 2021) e com desfecho completo nos estudos. Os critérios para a exclusão seriam: artigo duplicados, inferiores ao ano de 2011, artigos indisponíveis, estudos piloto, livros, capítulos de livros, revisões de literatura, estudos in vitro, teses e dissertações, editoriais, relatos de casos, artigos que após leitura não estava diretamente ligado à temática proposta ou que estavam relacionados ao tratamento odontológico com LBI, mas não interligado aos processos de biomodulação, além de estudos sobre LBI em outras áreas da saúde que não eram a odontologia.

Todos os dados qualitativos e quantitativos coletados foram tabulados através do software Microsoft Excel 2013, sendo destacados informações como o nome do autor/ano de publicação, materiais utilizados, tamanho da amostra, nível e duração do laser, resultados obtidos e desfecho do estudo (Quadro 1), o mesmo software foi utilizado para elaboração de gráficos contendo o total de estudos selecionados por ano de publicação (Gráfico 1) e os campos de estudos dentro da odontologia que estiveram mais prevalentes (Gráfico 2), além do fluxograma metodológico da pesquisa (Figura 1).

Figura 1 - Fluxograma PRISMA da pesquisa bibliográfica

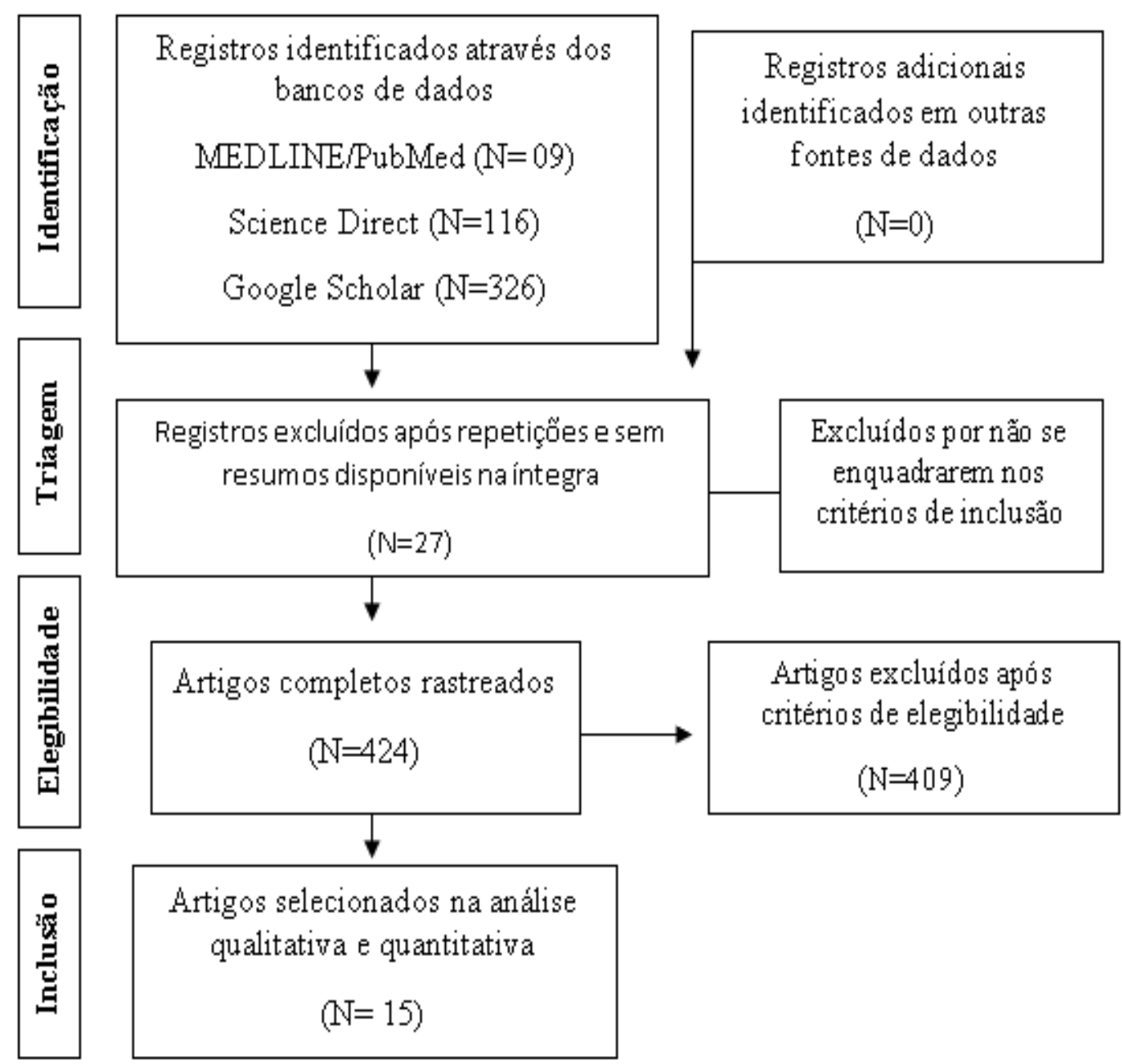

Fonte: Nogueira JES, et al., 2021. 


\section{RESULTADOS}

Com a busca na MEDLINE/PubMed, Science Direct e Google Scholar, encontrou-se 450 estudos na soma total das 3 plataformas. 27 estudos foram removidos devido a presença de itens duplicados, restando 424. Após a aplicação dos critérios de inclusão e exclusão adotados para esta revisão, descartou-se 409 artigos, restando apenas 15 estudos que ao final da leitura minuciosa foram incluídos. A quantidade de artigos selecionados por ano de publicação está destacada no Gráfico 1.

Gráfico 1 - Total de estudos selecionados por ano de publicação.

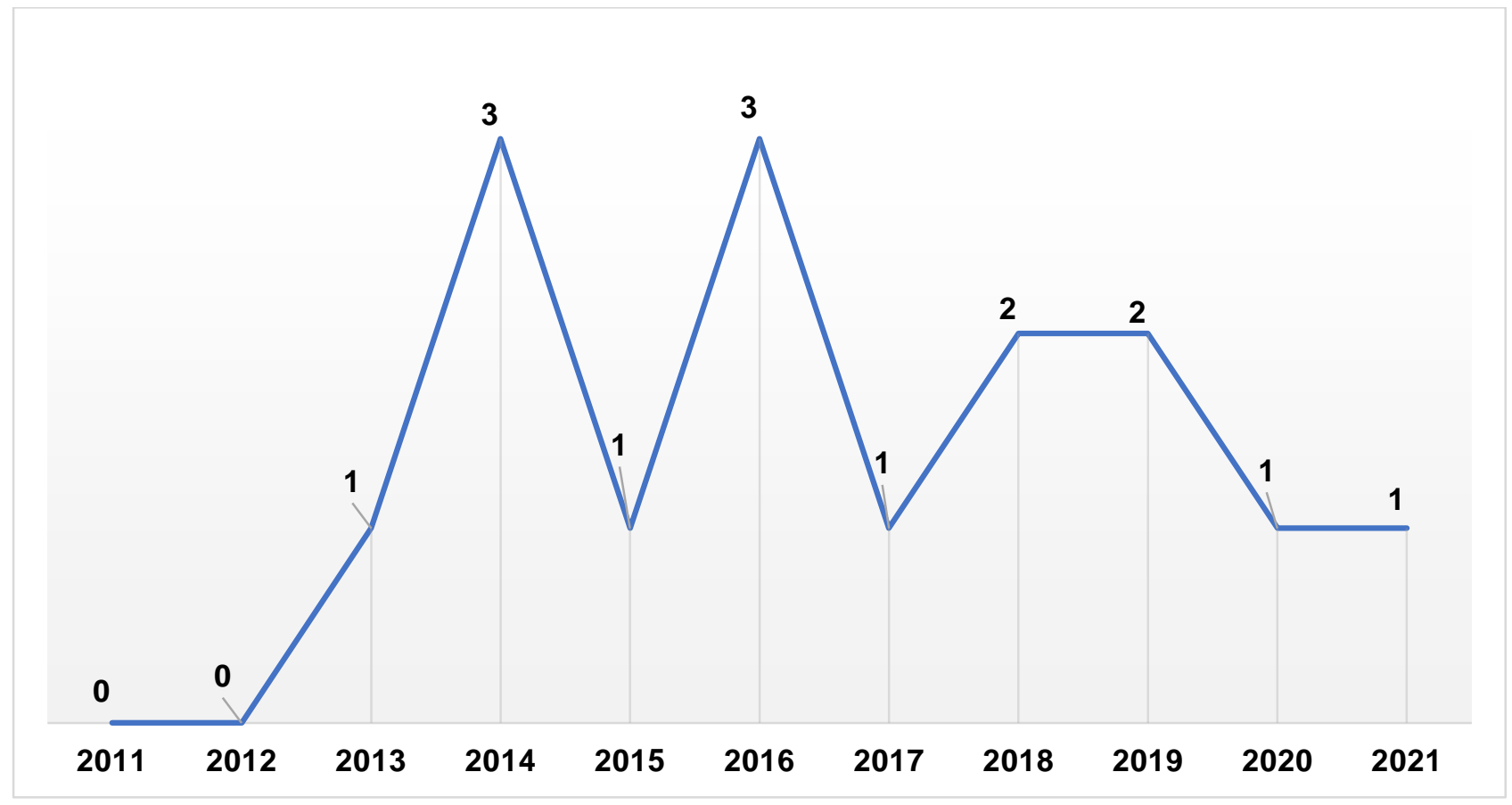

Fonte: Nogueira JES, et al., 2021.

Observa-se que houve uma maior prevalência no campo de estudo no ano de 2014 e 2016, totalizando 3 estudos, seguido de 2018 e 2019 ambos com 2 estudos. Dos 15 artigos selecionados, 14 eram ensaios clínicos e 1 pré-clínico. Tais pesquisas incluíram um total de 705 amostras na soma total dos estudos incluídos. Os autores dividiram as amostras em 2 a 5 grupos avaliativos, com no mínimo 10 e máximo 24 amostras em cada grupo teste ou controle.

No Quadro 1, observam-se as variáveis referente ao tratamento em estudo pré-clínicos e clínicos com LBI, número amostrais em cada estudo, parâmetros clínicos do laser como também a frequência dos de mediadores envolvidos dentro de cada campo de estudos com terapia e seu desfecho. Todos os estudos demonstraram uma resposta positiva no tratamento com LBI, auxiliando no processo de cicatrização, efeito anti-inflamatório, movimentação ortodôntica e reparo tecidual. 


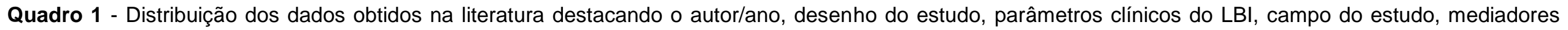
envolvidos e desfecho da terapia a laser.

\begin{tabular}{|c|c|c|c|c|c|}
\hline $\begin{array}{l}\text { Autor/ ano de } \\
\text { publicação }\end{array}$ & $\begin{array}{l}\text { Amostral } \\
(\mathrm{N})\end{array}$ & Parâmetros clínicos da LBI & $\begin{array}{l}\text { Campo do } \\
\text { estudo }\end{array}$ & $\begin{array}{l}\text { Citocinas e } \\
\text { fatores de } \\
\text { crescimento }\end{array}$ & Desfecho do ensaio \\
\hline $\begin{array}{l}\text { ABIDI AH, et al., } \\
2021 .\end{array}$ & $\mathrm{N}=96$ & $\begin{array}{l}\text { Diodo, } 660 \mathrm{~nm}, 810 \mathrm{~nm} \text { ou } 980 \\
\mathrm{~nm}, 200 \mathrm{~mW}, 0,32 \mathrm{~cm} 2,0,625 \mathrm{~W} / \\
\mathrm{cm} 2 .\end{array}$ & Periodontia & $\begin{array}{l}\text { LPS, TNF- } \alpha \text { e IL- } \\
1 \beta\end{array}$ & $\begin{array}{l}\text { Regulação negativa de mediadores inflamatórios + } \\
\text { LBI mostra-se promissora no tratamento da } \\
\text { inflamação periodontal. }\end{array}$ \\
\hline $\begin{array}{l}\text { CHEN YH, et al., } \\
2020 .\end{array}$ & $\mathrm{N}=19$ & $\begin{array}{l}\text { Diodo, } 660 \mathrm{~nm} \text { com uma } \\
\text { densidade de energia unilateral } \\
\text { de } 10 \mathrm{~J} / \mathrm{cm} 2 \text {. }\end{array}$ & Periodontia & $\begin{array}{l}\mathrm{IL}-1 \beta \text { e MMP-8 do } \\
\text { fluido gengival }\end{array}$ & $\begin{array}{l}\text { IL-1 } 1 \beta \text { e MMP- } 8 \text { foram reduzidos em todos os grupos } \\
\text { avaliados. }\end{array}$ \\
\hline $\begin{array}{l}\text { ANGIERO F, et } \\
\text { al., } 2019 .\end{array}$ & $\mathrm{N}=40$ & $\begin{array}{l}\text { Diodo, } 645 \mathrm{~nm}, 10 \mathrm{~J} / \mathrm{cm} 2 \text { e } 0,5 \mathrm{~W} \\
/ \mathrm{cm} 2,600 \mu \mathrm{m} .\end{array}$ & Periodontia & BK, VEGF, EGF & $\begin{array}{l}\text { Efeitos positivos do LBI nas fases iniciais do } \\
\text { processo de cicatrização, desempenhando um } \\
\text { papel na modulação de BK, EGF e VEGF nos níveis } \\
\text { de fluido de fendas gengivais }\end{array}$ \\
\hline $\begin{array}{l}\text { REN C, et al., } \\
2019 .\end{array}$ & $\mathrm{N}=27$ & $\begin{array}{l}\text { Diodo, } 940 \mathrm{~nm}, 2,8 \mathrm{~cm} \mathrm{2,} \mathrm{8,6} \mathrm{J} \mathrm{/} \mathrm{cm} \\
2 .\end{array}$ & Ortodontia & $\begin{array}{l}\mathrm{IL}-1 \beta \\
\text { prostaglandina } E \\
\text { 2, substância } P\end{array}$ & $\begin{array}{l}\text { LBI inibiu a elevação da interleucina- } 1 \beta, 2 \text { e níveis } \\
\text { de substância } P \text {, aliviando a dor e controlando a } \\
\text { inflamação periodontal durante o estágio inicial do } \\
\text { tratamento ortodôntico. }\end{array}$ \\
\hline $\begin{array}{l}\text { YĐLDĐZ ED, et } \\
\text { al., } 2018 .\end{array}$ & $\mathrm{N}=26$ & Diodo $970,15 \mathrm{~nm}, 0,5 \mathrm{~W}$ e $10 \mathrm{~Hz}$ & Endodontia & Substância P & $\begin{array}{l}\text { LBI possui efeito de imunomodulação ligado à } \\
\text { modulação da quantidade total da substância } P \text { no } \\
\text { fluido gengival das fendas. }\end{array}$ \\
\hline $\begin{array}{l}\text { VARELLA AM, et } \\
\quad \text { al., } 2018 .\end{array}$ & $\mathrm{N}=10$ & Diodo, 940nm, $8 \mathrm{~J} / \mathrm{cm} 2,100 \mathrm{~mW}$. & Ortodontia & IL-1 $\beta$ & $\begin{array}{l}\text { LBI aumentou os níveis de } \mathrm{IL}-1 \beta \text { e promoveu } \\
\text { aceleração ortodôntica. }\end{array}$ \\
\hline $\begin{array}{l}\text { SALVADOR } \\
\text { DRN, et al., } 2017 .\end{array}$ & $\mathrm{N}=51$ & $\begin{array}{l}\text { Diodo, } 660 \mathrm{~nm}, 40 \mathrm{~mW}, 0,16 \mathrm{~J}, 4 \mathrm{~J} \\
\text { / cm2. }\end{array}$ & Mucosite Oral & $\begin{array}{l}\text { CXCL8, IL-8ß, } \\
\text { nitrito e } \\
\text { mieloperoxidase }\end{array}$ & $\begin{array}{l}\text { LBI gerou uma redução na gravidade da } \mathrm{MO} \text {, a } \\
\text { redução foi associada a diminuição nos níveis } \\
\text { salivares de CXCL8. }\end{array}$ \\
\hline $\begin{array}{l}\text { EKIZER A, et al., } \\
2016 .\end{array}$ & $\mathrm{N}=20$ & $\begin{array}{l}\text { Diodo, } 618 \mathrm{~nm}, 20 \mathrm{~mW} / \mathrm{cm}, 20 \\
\text { minutos. }\end{array}$ & Ortodontia & IL-1 $\beta$ & $\begin{array}{l}\text { Nenhuma mudança estatisticamente significativa foi } \\
\text { detectada nos níveis de IL-1 } 1 \beta \text { entre os grupos. }\end{array}$ \\
\hline
\end{tabular}




\begin{tabular}{|c|c|c|c|c|c|}
\hline $\begin{array}{l}\text { Autor/ ano de } \\
\text { publicação }\end{array}$ & $\begin{array}{l}\text { Amostral } \\
\quad(\mathrm{N})\end{array}$ & Parâmetros clínicos da LBI & $\begin{array}{l}\text { Campo do } \\
\text { estudo }\end{array}$ & $\begin{array}{l}\text { Citocinas e } \\
\text { fatores de } \\
\text { crescimento }\end{array}$ & Desfecho do ensaio \\
\hline $\begin{array}{l}\text { THEODORO LH, } \\
\text { et al., } 2016 .\end{array}$ & $N=120$ & $\begin{array}{l}\text { Diodo, } 660 \mathrm{~nm} ; 0,035 \mathrm{~W} ; 4,2 \mathrm{~J} \\
120 \mathrm{~s} .\end{array}$ & Periodontia & $\begin{array}{l}\text { Prostaglandina } \\
\text { E2, TNF- } \alpha \text { e a IL- } \\
6 \beta\end{array}$ & $\begin{array}{l}\text { Grupo } 5 \text { FU-SRP-4-LBI apresentou menor ABL em } \\
\text { comparação com o grupo } 5 \text { FU no dia } 7 \text { e diminuiu } \\
\text { Imunomarcação de RANKL. }\end{array}$ \\
\hline $\begin{array}{l}\text { KUMARESAN D, } \\
\text { et al., } 2016 .\end{array}$ & $\mathrm{N}=90$ & Diodo, 810 nm, 0,7 W, $20 \mathrm{~s}$. & Periodontia & Periostina & $\begin{array}{l}\text { LBI apresentou benefícios adicionais sobre a DSR } \\
\text { nos parâmetros clínicos periodontais e aos níveis de } \\
\text { periostina do GCF }\end{array}$ \\
\hline $\begin{array}{l}\text { OTON-LEITE AF, } \\
\text { et al., } 2015 .\end{array}$ & $N=30$ & $\begin{array}{l}\text { Diodo, } 660 \mathrm{~nm}, 25 \mathrm{~mW}, 6,2 \mathrm{~J} \mathrm{/} \\
\mathrm{cm} 2 \text {, energia por ponto de 0,24 J. }\end{array}$ & Mucosite Oral & $\begin{array}{l}\text { TNF- } \alpha, \text { IL-6, IL- } \\
1 \beta, \text { IL-10, TGF- } \beta \text { ), } \\
\text { EGF, FGF, VEGF } \\
\text { e MMP2 / TIMP2, } \\
\text { MMP9 / TIMP }\end{array}$ & $\begin{array}{l}\text { LBI eficaz na redução da gravidade da } \mathrm{MO} \text { e foi } \\
\text { associada à redução da inflamação e do reparo. }\end{array}$ \\
\hline $\begin{array}{l}\text { SILVA GBL, et } \\
\text { al., } 2014 .\end{array}$ & $N=30$ & $\begin{array}{l}\text { Diodo, luz contínua a } 660 \mathrm{~nm}, 0 \\
\mathrm{~mW}, 0,16 \mathrm{~J}, 4 \mathrm{~J} / \mathrm{cm} 2 \text {. }\end{array}$ & Mucosite Oral & $\begin{array}{l}\text { TNF- } \alpha, \text { IL- } 6, \text { IL- } \\
1 \beta, \text { IL-10, TGF- } \beta \text {, } \\
\text { MMP, e fatores } \\
\text { de crescimento }\end{array}$ & $\begin{array}{l}\mathrm{LBI} \text { eficaz na redução da } \mathrm{MO} \text {. O mecanismo de } \\
\text { ação pode não estar completamente associado a } \\
\text { determinados processos moleculares. }\end{array}$ \\
\hline $\begin{array}{l}\text { SAGLAM M, et } \\
\text { al., } 2014 .\end{array}$ & $\mathrm{N}=30$ & Diodo, 1,5 W, 15 J / cm2, $300 \mu \mathrm{m}$. & Periodontia & $\begin{array}{l}\text { IL-1 } 1 \beta, \text { IL-6, IL-8, } \\
\text { MMP-1, MMP- } 8 \\
\text { TIMP-1 }\end{array}$ & $\begin{array}{l}\text { MMP-8 foi significativamente afetada pelo LBI, } \\
\text { melhorando os resultados da terapia periodontal não } \\
\text { cirúrgica. }\end{array}$ \\
\hline $\begin{array}{l}\text { YOO YJ, et al., } \\
2014 .\end{array}$ & $\mathrm{N}=40$ & Nd: YAG, 1440 nm,300 $\mu \mathrm{m}$. & Periodontia & $\begin{array}{l}\text { Substância P, } \\
\text { CGRP e MMP - } 8\end{array}$ & $\begin{array}{l}\text { LBI melhorou o alívio da dor na percussão e na } \\
\text { redução dos níveis de substância } P \text {, CGRP e MMP- } \\
8 \text {. }\end{array}$ \\
\hline $\begin{array}{l}\text { WU JY, et al., } \\
2013 .\end{array}$ & $N=96$ & Diodo, $660 \mathrm{~nm}, 0,1,2$ ou $4 \mathrm{~J} \cdot \mathrm{cm}$ & Periodontia & cAMP & $\begin{array}{l}\text { LBI promoveu aumento de proliferação e } \\
\text { diferenciação osteogênica de células HPDL. }\end{array}$ \\
\hline
\end{tabular}

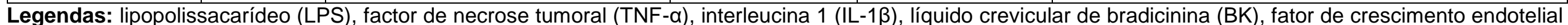

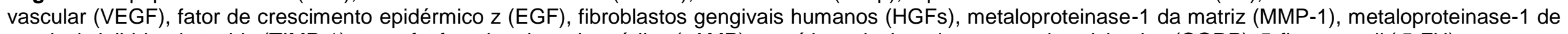

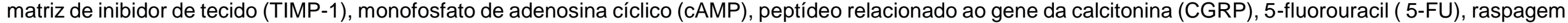

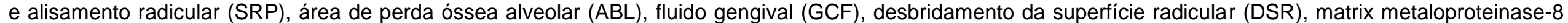
(MMP-8), Watts (W), Megawatts (mW), joules (J), fator de crescimento fibroblástico (FGF).

Fonte: Nogueira JES, et al., 2021. 
A maioria dos estudos estavam relacionados a terapia a laser de baixa intensidade em avaliação a doenças periodontais, com um total de 8 estudos (53\%), sendo 7 ensaios clínicos e 1 pré-clínico. A mucosite oral associada a LBI, e ortodontia também tiveram 3 estudos avaliado cada ( $20 \%$ cada) e com menor prevalência a endodontia com apenas um estudo (7\%), conforme ilustrado no Gráfico 2.

Gráfico 2- Campo de estudos prevalente na literatura

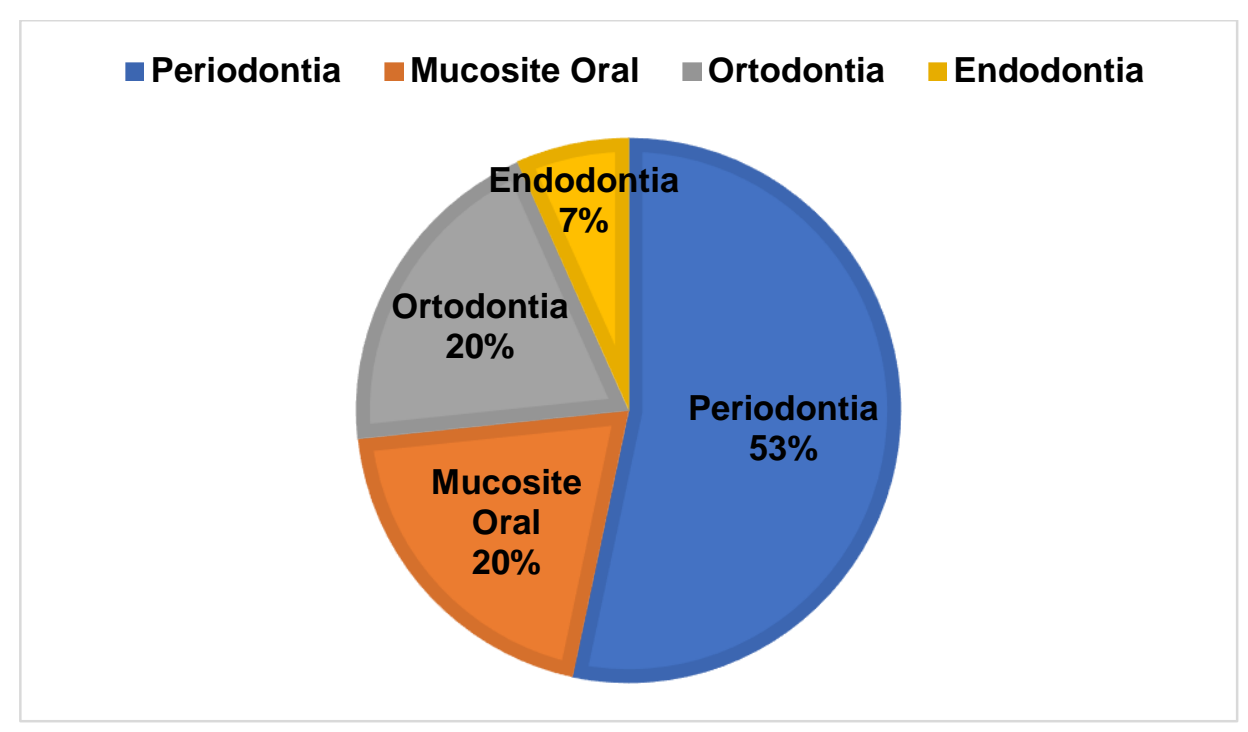

Fonte: Nogueira JES, et al., 2021.

\section{DISCUSSÃO}

A literatura demonstra essa terapia como promissora em termos de aplicabilidade clínica, evidenciando respostas modulares em sua ação que promovem uma melhor regeneração celular em comparação a métodos farmacológicos na odontologia. É recomendada por alguns pesquisadores devido seus efeitos na redução da dor, efeitos anti-inflamatórios e cicatrizantes (ANGIERO F, et al., 2019). Seu comprimento de onda mais curtos pode variar de 532 a 1064 nanômetros $(\mathrm{nm})$ e são melhores absorvidos por moléculas pigmentadas como hemoglobina e melanina, enquanto comprimentos de onda mais longos variam de 3.000 $\mathrm{nm}$ e $10.600 \mathrm{~nm}$ e estes experimentam melhor interação com a água e conteúdo mineral do tecido (SRIVASTAVA V, et al., 2014).

A LBI envolve o contato com tecidos inflamados visando eliminar a infecção, interromper a progressão da doença além de regenerar o periodonto comprometido (ANGIERO $F$, et al., 2019). Em tratamentos odontológicos pode demonstrar resultados variáveis dependendo de seu comprimento e duração de aplicação. Em relação a estudos pré-clínicos com a LBI e sua análise em processos moleculares, Theodoro $\mathrm{LH}$, et al. (2016) relatam em seu estudo que os grupos de camundongos tratados com 5 -fluorouracil (5FU) apresentaram maior área de perda óssea alveolar ( $\mathrm{ABL}$ ) em comparação com o grupo controle, os autores evidenciam também que o 5-FU agravou a periodontite, apesar disso, várias sessões da LBI associada à raspagem e alisamento radicular (SRP) melhoraram a periodontite nos animais testados.

Já nos ensaios clínicos no campo da periodontia, Kumaresan D, et al. (2016), destacam que os níveis de periostina foram significativamente menores em pacientes com periodontite crônica (PC) quando levado em comparação a indivíduos saudáveis. Similiar ao seu estudo, Abidi AH, et al. (2021) observaram que o tratamento com laser de $660 \mathrm{~nm}$ induziu citocinas pró-inflamatórias quando estimulado, sendo que o comprimento de onda de $810 \mathrm{~nm}$ exibiu sozinho efeitos anti-inflamatórios para todos os campos avaliados, exceto para IL-8. A regulação negativa de mediadores inflamatórios pela combinação ou tratamento individual com comprimento de onda de $810 \mathrm{~nm}$, assim como no estudo de Kumaresan D, et al. (2016), mostrou-se eficaz no tratamento da inflamação periodontal. 
Levando em consideração os efeitos biologicamente benéficos promovidos pela LBI, o uso dessa ferramenta durante o processo de regeneração do periodonto pode ser útil em termos terapêuticos (WU JY, et al., 2013). Assim, Yoo YJ, et al. (2014) tiveram desfechos similares ao de Abidi AH, et al. (2021) no qual evidenciaram que o laser Nd: YAG de $1440 \mathrm{~nm}$ teve um efeito significativamente melhor no alívio da dor na percussão e na redução dos níveis de substância peptídeo relacionado ao gene da calcitonina (CGRP) e MMP-8. Ligado a isso, Angiero F, et al. (2019) obteve um número amostral igual ao de Yoo YJ, et al. (2014), porém, seus resultados foram diferentes em termo de efetividade da ferramenta, mostrando que a LBI tem efeitos benéficos nas fases iniciais do processo de cicatrização, desempenhando ainda um papel na modulação de BK, EGF e VEGF nos níveis de fluido das fendas gengivais.

Apesar de alguns estudos demostrarem eficácia na aplicação da LBI em procedimentos clínicos na odontologia, outros não demostraram valores significativos nos grupos avaliativos. No campo da endodontia, pacientes sofrem em decorrência de vários níveis de dor e geralmente estão expressas como dor espontânea ou dor à percussão durante ou após o tratamento (YOO YJ, et al., 2014). Nesse viés, Yldiz ED, et al. (2019) relata que não houve diferença significantes entre as quantidades totais pré e pós-operatórias do nível de substância $P$, que segundo os autores, essa substância pode ser definida como um neurotransmissor envolvido no aumento de respostas inflamatórias. Seus resultados foram divergentes aos de Ren $\mathrm{C}$, et al. (2020), no qual a irradiação o laser inibiu a elevação da interleucina-1 $\beta$, 2 e também nos níveis de substância $P$ durante o primeiro mês de estudo.

Os lasers possuem classificação de acordo com o comprimento de onda, sistema de entrega das dosagens, modos em que será emitido, absorção no tecido alvo, além das aplicações clínicas da ferramenta (SAGLAM M, et al., 2014). Diferentemente dos lasers cirúrgicos que necessitam de mais de 1 watts sendo que a potência desses lasers pode ser alterada para que se possa obter parâmetros como a fluência e densidade de potência adequada, com comprimentos de onda geralmente no espectro vermelho e infravermelho (ANGIERO F, et al., 2019).

Promove diversas respostas no organismo, e sua ação modeladora pode variar conforme a sua utilização clínica. Sobre isso, Wu JY, et al. (2013) observaram que a LBI promoveu em valores significativos a proliferação de células do ligamento periodontal humano (HPDL). A ferramenta mostrou ainda potencial capacidade osteogênica tendo em vista a estimulação da atividade de fosfatase alcalina (ALP) em seu estudo, além da deposição de cálcio e expressão de genes osteogênicos.

Além da periodontia e endodontia, a LBI pode ser utilizada em outros campos da odontologia. Assim, as aplicações dos lasers no campo da ortodontia são diversificadas e o seu uso vai desde o diagnóstico como a varredura da superfície, anomalias craniofaciais, até a gravação em processos de colagem eficientes (SRIVASTAVA V, et al., 2014).

Varella AM, et al. (2018) observaram em seu estudo níveis aumentados de IL-1 $\beta$ nos dentes caninos experimentais quando comparados aos caninos no grupo controle, além de haver uma correlação positiva entre os níveis de IL-1 $\beta$ e a quantidade de movimento dentário em todos os intervalos de tempo avaliados. Neste estudo, a combinação de força ortodôntica a aplicação da terapia a laser de baixa intensidade aumentou os níveis de $\mathrm{IL}-1 \beta$ no fluido das fendas gengivais e acelerou a movimentação dentária ortodôntica. Em contrapartida ao seu estudo, Ekizer A, et al. (2016) não obteve valores significativos nos níveis de IL-1 $\beta$ entre os grupos avaliados em um mesmo campo de estudo, o que sugere que diferentes parâmetros clínicos do laser em ambos os estudos puderam interferir em dados igualitários.

Dor e desconforto costumam ser as queixas dos pacientes durante tratamentos na odontologia, assim, os lasers podem promover o alívio para essas condições clínicas (SRIVASTAVA V, et al., 2014). Várias citocinas desempenham papéis de suma relevância na cicatrização de feridas como na inflamação, proliferação e regeneração tecidual no qual ocorrem após tratamentos odontológicos, mas relativamente poucos ensaios clínicos investigaram esses parâmetros, nem os benefícios da LBI utilizado em associação a outras terapias (ANGIERO F, et al., 2019). 
Visando avaliar esses fatores, Oton-Leite AF, et al. (2015), analisaram citocinas pró-inflamatórias e antiinflamatórias, fatores de crescimento e metaloproteinases. No estudo, o grupo laser apresentou redução da gravidade da $\mathrm{MO}$, seguindo com diminuição da concentração salivar de EGF e VEGF na sétima sessão de radioterapia e de IL-6 e FGF na 35a sessão. Houve tendência de redução dos níveis de citocinas próinflamatórias, anti-inflamatórias e fatores de crescimento no grupo laser em relação ao controle.

Em concordância a Oton-Leite AF, et al. (2015), o estudo de Silva GBL, et al. (2014) também buscou avaliar citocinas pró-inflamatórias e anti-inflamatórias, fatores de crescimento e metaloproteinases em pacientes tratados com quimioterapia, obtendo desfechos similares no qual também houve uma redução da MO com a utilização da LBI, mas que diferente de Oton-Leite et al. (2015), essa resposta do laser pode não estar totalmente associada a esses fatores biomoleculares, tendo em vista os achados significativos de seu ensaio clínico, apesar do grupo LBI ter apresentado aumento dos níveis de matriz metaloproteinase 2 (MMP2) na saliva.

O uso de lasers na área odontológica tem sido objeto de numerosos estudos pelos pesquisadores (SAGLAM M, et al., 2012), seu processo envolve eventos celulares e moleculares que podem ser associados a cicatrização de feridas (ANGIERO F, et al., 2019). Sobre isso, no estudo de Salvador DRN, et al. (2017) a LBI levou a uma diminuição nos níveis de interleucina 8 (CXCL8) em $85 \%$ dos pacientes tratados com a ferramenta em seu estudo, enquanto $70,8 \%$ dos pacientes do grupo controle apresentaram aumento dessa quimiocina. Além disso, a fermenta gerou uma redução na gravidade da $\mathrm{MO}$ em pacientes submetidos ao transplante de células-tronco hematopoiéticas, no qual este achado foi associado a uma diminuição nos níveis salivares de CXCL8.

Seus resultados foram similares aos de Chen YW, et al. (2020) no qual evidenciaram que também houve uma redução nos níveis de CXCL8 em seu estudo, sendo que essa redução foi mais presentes no grupo tratado com raspagem e planejamento radicular, o que difere do estudo de Saglam M, et al. (2012) no qual evidenciaram melhoras na terapia periodontal, principalmente relacionada ao aumento nos níveis de CXCL8 em associação ao LBI. O processo celular e molecular em associação ao LBI pode gerar diversas respostas internas que podem variar conforme a terapia preemptiva utilizada antes dos procedimentos, como raspagem e alisamento radicular. Os benefícios da abordagem da LBI ainda precisam ser estabelecidos de maneira mais analítica (ANGIERO F, et al., 2019).

Desse modo, observa-se que ainda não existe um consenso sobre os resultados de tratamento com essa ferramenta, principalmente resultados a longos prazos no qual são desconhecidos na literatura (SAGLAM M, et al., 2012). Assim, o mecanismo pelo qual a LBI promove uma resposta celular e as condições ideais para essa terapia, como a fluência e o comprimento de onda ainda não estão claras cientificamente (WU JY, et al., 2013).

\section{CONSIDERAÇÕES FINAIS}

Através dos dados obtidos pela literatura, é possível evidenciar a LBI como uma ferramenta promissora na odontologia, promovendo diversas respostas fisiológicas, como a regeneração celular de maneira mais rápida quando levada em comparação a outras formas terapêuticas avaliadas nos estudos, além de movimentação ortodôntica e reparos periodontais. O estudo pode contribuir como complemento no conhecimento de cirurgiões-dentistas nos processos inflamatórios e celulares em procedimentos odontológicos com a LBI, sob diferentes mediadores e mecanismos que são empregados, auxiliando para um melhor entendimento e possibilitando assim a seleção da melhor terapêutica a ser empregada clinicamente. Dentre as limitações do estudo, observou-se uma escassez na literatura de ensaios clínicos que avaliem o processo de biomodulação da LBI em demais áreas da odontologia, como na Disfunção Temporomandibular (DTM) e cirurgia oral, o que sugere que mais estudos sejam realizados visando obter dados mais precisos sobre a aplicabilidade clínica desta ferramenta. 


\section{REFERÊNCIAS}

1. $A B I D I A H$, et al. Immunomodulatory activity seen as a result of photobiomodulation therapy in stimulated primary human fibroblasts. Archives Of Oral Biology, 2021; 121(10): 49-68.

2. ANGIERO F, et al. Evaluation of bradykinin, VEGF, and EGF biomarkers in gingival crevicular fluid and comparison of photoBioModulation with conventional techniques in periodontitis: a split-mouth randomized clinical trial. Lasers In Medical Science, 2019; 35(4): 965-970

3. ASSIS TO, et al. O uso do laser na reabilitação das desordens temporomandibulares. Fisioter. mov., Curitiba, 2012; 25(2): 453-459.

4. CHEN YW, et al. Randomized controlled clinical effectiveness of adjunct 660-nm light-emitting diode irradiation during non-surgical periodontal therapy. Journal Of The Formosan Medical Association, 2020; 119(1): 157-163.

5. EKIZER A, et al. Light emitting diode mediated photobiomodulation therapy improves orthodontic tooth movement and miniscrew stability: a randomized controlled clinical trial. Lasers In Surgery And Medicine, 2016; 48(10): 936-943.

6. JúNIOR C, et al. Laser terapêutico e células inflamatórias - parte 2: neutrófilos. Rev. Cir. Traumatol. Buco-MaxiloFac., 2013; 13(3): 111-118.

7. OTON-LEITE AF, et al. Effect of low-level laser therapy on chemoradiotherapy-induced oral mucositis and salivary inflammatory mediators in head and neck cancer patients. Lasers In Surgery And Medicine, 2015; 47(4): $296-305$.

8. THEODORO LH, et al. Effect of low-level laser therapy as an adjuvant in the treatment of periodontitis induced in rats subjected to 5-fluorouracil chemotherapy. Journal Of Periodontal Research, 2016; 51(5): 669-680.

9. KUMARESAN D, et al. Gingival crevicular fluid periostin levels in chronic periodontitis patients following nonsurgical periodontal treatment with low-level laser therapy. European Journal Of Dentistry, 2016; 10(4): 546-550.

10. REN C, et al. Low-level laser-aided orthodontic treatment of periodontally compromised patients: a randomised controlled trial. Lasers In Medical Science, 2019; 35(3): 729-739.

11. SANT'ANNA EF, et al. High-intensity laser application in Orthodontics. Dental Press J. Orthod., 2017; 22(6): 99-109.

12. SALVADOR DRN, et al. Effect of photobiomodulation therapy on reducing the chemo-induced oral mucositis severity and on salivary levels of CXCL8/interleukin 8, nitrite, and myeloperoxidase in patients undergoing hematopoietic stem cell transplantation: a randomized clinical trial. Lasers In Medical Science, 2017; 32(8): 1801-1810.

13. SAGLAM M, et al. Clinical and biochemical effects of diode laser as an adjunct to nonsurgical treatment of chronic periodontitis: a randomized, controlled clinical trial. Lasers In Medical Science, 2012; 29(1): 37-46.

14. SILVA GBL, et al. Effect of low-level laser therapy on inflammatory mediator release during chemotherapy-induced oral mucositis: a randomized preliminary study. Lasers In Medical Science, 2014; 30(1): 117-126.

15. SOUZA ACOC, et al. A influência da laserterapia de baixa potência e do ultrassom terapêutico na abertura da boca em pacientes com disfunção temporomandibular. Revista Artigos. Com, 2020; 22: e6006.

16. SOUZA MT, et al. Revisão integrativa: o que é e como fazer. Einstein, São Paulo, 2010; 8(1): 102-106.

17. SOUZA NHC, et al. Efeito da laserterapia em baixa intensidade na modulação da atividade mitocondrial de macrófagos. Braz. J. Phys. Ther., 2014; 18(4): 308-314.

18. SRIVASTAVA V, et al. Diode lasers: a magical wand to an orthodontic practice. Indian Journal Of Dental Research, $2014 ; 25(1): 78-82$.

19. YĐLDĐZ ED, et al. The effect of photobiomodulation on total amount of substance $P$ in gingival crevicular fluid: placebocontrolled randomized clinical trial. Lasers In Medical Science, 2018; 34(3): 517-523.

20. YOO YJ, et al. Effect of 1440-Nanometer Neodymium: yttrium-aluminum-garnet laser irradiation on pain and neuropeptide reduction. Journal Of Endodontics, 2014; 40(1): 28-32.

21. VARELLA AM, et al. Low-level laser therapy increases interleukin-1 $\beta$ in gingival crevicular fluid and enhances the rate of orthodontic tooth movement. American Journal Of Orthodontics And Dentofacial Orthopedics, 2018; 154(4): 535544.

22. WU JY, et al. Low-power laser irradiation promotes the proliferation and osteogenic differentiation of human periodontal ligament cells via cyclic adenosine monophosphate. International Journal Of Oral Science, 2013; 5(2): 85-91. 